\title{
Approximate controllability of fractional impulsive neutral stochastic differential equations with nonlocal conditions
}

\section{Yanchao Zang ${ }^{*}$ and Junping Li}

\section{"Correspondence:} zycmail@126.com

School of Mathematics and Statistics, Central South University, Changsha, Hunan 410075, P.R. China

\begin{abstract}
In this paper, the approximate controllability of fractional impulsive neutral stochastic differential equations with nonlocal conditions and infinite delay in Hilbert spaces is studied. By using the Krasnoselskii-Schaefer-type fixed point theorem and stochastic analysis theory, some sufficient conditions are given for the approximate controllability of the system. At the end, an example is given to illustrate the application of our result.
\end{abstract}

MSC: 65C30; 93B05; 34K40; 34K45

Keywords: approximate controllability; fixed point principle; fractional impulsive neutral stochastic differential equations; mild solution; nonlocal conditions

\section{Introduction}

The purpose of this paper is to prove the existence and approximate controllability of mild solutions for a class of fractional impulsive neutral stochastic differential equations with nonlocal conditions described in the form

$$
\left\{\begin{aligned}
&{ }^{c} D_{t}^{\alpha}\left[x(t)-h\left(t, x_{t}\right)\right]= A\left[x(t)-h\left(t, x_{t}\right)\right]+B u(t)+f\left(t, x_{t}\right) \\
&+g\left(t, x_{t}\right) \frac{d W(t)}{d t}, \quad t \in J:=[0, T], \\
&\left.\Delta x\right|_{t=\tau_{k}}=I_{k}\left(x\left(\tau_{k}^{-}\right)\right), \quad k=1,2, \ldots, n, \\
& x(0)+\mu(x)=x_{0}=\varphi \in \mathcal{C}_{v},
\end{aligned}\right.
$$

where ${ }^{c} D_{t}^{\alpha}$ is the Caputo fractional derivative of order $\frac{1}{2}<\alpha<1$; the state variable $x(\cdot)$ takes values in the real separable Hilbert space $H ; A: D(A) \subset H \rightarrow H$ is the infinitesimal generator of a strongly continuous semigroup of a bounded linear operators $T(t), t \geq 0$, in the Hilbert space $H$. The history $x_{t}:(-\infty, 0] \rightarrow H, x_{t}(\theta)=x(t+\theta), \theta \leq 0$, belongs to an abstract phase space $\mathcal{C}_{v}$. The control function $u(\cdot)$ is given in $L^{2}(J, U), U$ is a Hilbert space; $B$ is a bounded linear operator from $U$ into $H$. The functions $f, h, g, I_{k}$ are appropriate functions to be specified later. The process $\{W(t): t \geq 0\}$ is a given $U$-valued Wiener process with a finite trace nuclear covariance operator $Q \geq 0$ defined on a complete probability space $\left(\Omega, \mathcal{F},\left\{\mathcal{F}_{t}\right\}, P\right)$. Here $0 \leq \tau_{1} \leq \cdots \leq \tau_{n} \leq T,\left.\Delta x\right|_{t=\tau_{k}}=x\left(\tau_{k}^{+}\right)-x\left(\tau_{k}^{-}\right)$, $x\left(\tau_{k}^{+}\right)$and $x\left(\tau_{k}^{-}\right)$represent the right and left limits of $x(t)$ at $t=\tau_{k}$, respectively. The initial data $\varphi=\{\varphi(t), t \in(-\infty, 0]\}$ is an $\mathcal{F}_{0}$-measurable, $\mathcal{C}_{v}$-valued random variable independent of $W(t)$ with finite second moments.

(c) 2013 Zang and Li; licensee Springer. This is an Open Access article distributed under the terms of the Creative Commons Attribution License (http://creativecommons.org/licenses/by/2.0), which permits unrestricted use, distribution, and reproduction in any medium, provided the original work is properly cited. 
In the past few decades, the theory of fractional differential equations has received a great deal of attention, and they play an important role in many applied fields, including viscoelasticity, electrochemistry, control, porous media, electromagnetic and so on. We refer the reader to the monographs of Kilbas et al. [1], Mill and Ross [2], Podlubny [3] and the references therein. There is also an extensive literature concerned with the fractional differential equations. For example, Benchohra et al. in [4] considered the VIP for a particular class of fractional neutral functional differential equations with infinite delay. Zhou in [5] discussed the existence and uniqueness for fractional neutral differential equations with infinite delay.

In practice, deterministic systems often fluctuate due to environmental noise. So it is important and necessary for us to discuss the stochastic differential systems. On the other hand, the control theory is one of the important topics in mathematics. Roughly speaking, controllability generally means that it is possible to steer a dynamical control system from an arbitrary initial state to an arbitrary final state using the set of admissible controls. As a result of its widespread use, the controllability of stochastic or deterministic systems all have received extensive attention. Mahmudov [6] investigated the controllability of infinite dimensional linear stochastic systems, and in [7] Dauer and Mahmudov extended the results to semilinear stochastic evolution equations with finite delay. Park, Balasubramaniam and Kumaresan [8] gave the controllability of neutral stochastic functional infinite delay systems. Besides the environmental noise, sometimes, we have to consider the impulsive effects, which exist in many evolution processes, because the impulsive effects may bring an abrupt change at certain moments of time. For the literatures on controllability of stochastic system with impulsive effect, we can see [9-13].

However, to the best of our knowledge, it seems that little is known about approximate controllability of fractional impulsive neutral stochastic differential equations with infinite delay and nonlocal conditions. The aim of this paper is to study this interesting problem. The rest of the paper is organized as follows. In Section 2, we introduce some preliminaries such as definitions of fractional calculus and some useful lemmas. In Section 3, we prove our main results. Finally in Section 4, an example is given to demonstrate the application of our results.

\section{Preliminaries}

In this section, we introduce some notations and preliminary results, needed to establish our results. Throughout this paper, let $U$ and $H$ be two real separable Hilbert spaces, and we denote by $\mathcal{L}(U, H)$ the set of all linear bounded operators from $U$ into $H$. For convenience, we will use the same notation $\|\cdot\|$ to denote the norms in $U, H$ and $\mathcal{L}(U, H)$, and use $\langle\cdot, \cdot\rangle$ to denote the inner product of $U$ and $H$ without any confusion. Let $\left(\Omega, \mathcal{F},\left\{\mathcal{F}_{t}\right\}_{t \geq 0}, P\right)$ be a complete probability space with a filtration $\left\{\mathcal{F}_{t}\right\}_{t \geq 0}$ satisfying the usual conditions (i.e., it is increasing and right continuous, while $\mathcal{F}_{0}$ contains all $P$-null sets). Let $W=\left(W_{t}\right)_{t \geq 0}$ be a $Q$-Wiener process defined on $\left(\Omega, \mathcal{F},\left\{\mathcal{F}_{t}\right\}_{t \geq 0}, P\right)$ with the covariance operator $Q$, that is

$$
\mathbb{E}|W(t), x\rangle\langle W(s), y\rangle=(t \wedge s)\langle Q x, y\rangle \quad \text { for all } x, y \in U \text { and } t, s \in[0, T],
$$


where $Q$ is a positive, self-adjoint, trace class operator on $U$. Let $\mathcal{L}_{2}^{0}=\mathcal{L}_{2}(U, H)$ be the space of all $Q$-Hilbert-Schmidt operators from $U$ to $H$ with the norm

$$
\|\xi\|_{\mathcal{L}_{2}^{0}}^{2}:=\operatorname{tr}\left(\xi Q \xi^{*}\right)<\infty, \quad \xi \in \mathcal{L}(U, H)
$$

For the construction of stochastic integral in Hilbert space, see Da Prato and Zabczyk [14]. Let $A$ be the infinitesimal generator of an analytic semigroup $\{T(t)\}_{t \geq 0}$ of uniformly bounded linear operators on $H$, and in this paper, we always assume that $T(t)$ is compact.

Now, we present the abstract space $\mathcal{C}_{v}$. Assume that $v:(-\infty, 0] \rightarrow(0,+\infty)$ with $l=$ $\int_{-\infty}^{0} v(t) d t<+\infty$ is a continuous function. The abstract phase space $\mathcal{C}_{v}$ is defined by $\mathcal{C}_{v}=$ $\left\{\varphi:(-\infty, 0] \rightarrow H\right.$, for any $a>0,\left(E|\varphi(\theta)|^{2}\right)^{1 / 2}$ is a bounded and measurable function on $[-a, 0]$ and $\left.\int_{-\infty}^{0} v(s) \sup _{s \leq \theta \leq 0}\left(E|\varphi(\theta)|^{2}\right)^{1 / 2} d s<+\infty\right\}$. If $\mathcal{C}_{v}$ is endowed with the norm

$$
\|\varphi\|_{\mathcal{C}_{v}}=\int_{-\infty}^{0} v(s) \sup _{s \leq \theta \leq 0}\left(E|\varphi(\theta)|^{2}\right)^{1 / 2} d s, \quad \varphi \in \mathcal{C}_{v}
$$

then $\left(\mathcal{C}_{v},\|\cdot\|_{\mathcal{C}_{v}}\right)$ is a Banach space $[15,16]$.

Now, we consider the space

$$
\begin{aligned}
\mathcal{B}_{T}:= & \left\{x:(-\infty, T] \rightarrow H, x_{k} \in C\left(J_{k}, H\right) \text { and there exist } x\left(\tau_{k}^{-}\right) \text {and } x\left(\tau_{k}^{+}\right)\right. \\
& \text {with } \left.x\left(\tau_{k}\right)=x\left(\tau_{k}^{-}\right), x_{0}=\varphi \in \mathcal{C}_{v}, k=0,1,2, \ldots, n\right\},
\end{aligned}
$$

where $x_{k}$ is the restriction of $x$ to $J_{k}=\left(\tau_{k}, \tau_{k+1}\right], k=0,1,2, \ldots, n$. We endow a seminorm $\|\cdot\|_{\mathcal{B}_{T}}$ on $\mathcal{B}_{T}$, it is defined by

$$
\|x\|_{\mathcal{B}_{T}}=\|\varphi\|_{\mathcal{C}_{v}}+\sup _{s \in[0, T]}\left(E\|x(s)\|^{2}\right)^{1 / 2}, \quad x \in \mathcal{B}_{T}
$$

Lemma 2.1 (see [17]) Assume that $x \in \mathcal{B}_{T}$, then for $t \in J, x_{t} \in \mathcal{C}_{v}$. Moreover,

$$
l\left(E\|x(t)\|^{2}\right)^{1 / 2} \leq\left\|x_{t}\right\|_{\mathcal{C}_{v}} \leq l \sup _{s \in[0, t]}\left(E\|x(s)\|^{2}\right)^{1 / 2}+\left\|x_{0}\right\|_{\mathcal{C}_{v}},
$$

where $l=\int_{-\infty}^{0} v(s) d s<\infty$

Definition 2.1 The fractional integral of order $\alpha$ with the lower limit 0 for a function $f$ is defined as

$$
I^{\alpha} f(t)=\frac{1}{\Gamma(\alpha)} \int_{0}^{t} \frac{f(s)}{(t-s)^{1-\alpha}} d s, \quad t>0, \alpha>0
$$

provided the right side is pointwise defined on $[0, \infty)$, where $\Gamma(\cdot)$ is the gamma function.

Definition 2.2 The Caputo derivative of order $\alpha$ with the lower limit 0 for a function $f$ can be written as

$$
{ }^{c} D_{t}^{\alpha} f(t)=\frac{1}{\Gamma(n-\alpha)} \int_{0}^{t} \frac{f^{n}(s)}{(t-s)^{\alpha+1-n}} d s=I^{n-\alpha} f^{n}(t), \quad t>0, n-1<\alpha<n .
$$


Definition 2.3 A stochastic process $x: J \times \Omega \rightarrow H$ is called a mild solution of the system (1) if

(i) $x(t)$ is measurable and $\mathscr{F}_{t}$-adapted, for each $t \geq 0$;

(ii) $x(t) \in H$ has càdlàg paths on $t \in[0, T]$ a.s., and satisfies the following integral equation

$$
\begin{aligned}
x(t)= & \mathcal{T}(t)[\varphi(0)-\mu(x)-h(0, \varphi)]+h\left(t, x_{t}\right)+\int_{0}^{t}(t-s)^{\alpha-1} s(t-s) B u(s) d s \\
& +\int_{0}^{t}(t-s)^{\alpha-1} s(t-s) f\left(s, x_{s}\right) d s \\
& +\int_{0}^{t}(t-s)^{\alpha-1} s(t-s) g\left(s, x_{s}\right) d W(s) \\
& +\sum_{0<\tau_{k}<t} \mathcal{T}\left(t-\tau_{k}\right) I_{k}\left(x\left(\tau_{k}^{-}\right)\right), \quad t \in J ;
\end{aligned}
$$

(iii) $x_{0}(\cdot)=\varphi \in \mathcal{C}_{v}$ on $(-\infty, 0]$ satisfying $\|\varphi\|_{\mathcal{C}_{v}}<\infty$, where

$$
\begin{aligned}
& \mathcal{T}(t)=\int_{0}^{\infty} \xi_{\alpha}(\theta) T\left(t^{\alpha} \theta\right) d \theta, \quad \&(t)=\alpha \int_{0}^{\infty} \theta \xi_{\alpha}(\theta) T\left(t^{\alpha} \theta\right) d \theta, \\
& \xi_{\alpha}(\theta)=\frac{1}{\alpha} \theta^{-1-\frac{1}{\alpha}} \varpi_{\alpha}\left(\theta^{-\frac{1}{\alpha}}\right) \geq 0, \\
& \varpi_{\alpha}(\theta)=\frac{1}{\pi} \sum_{n=1}^{\infty}(-1)^{n-1} \theta^{-n \alpha-1} \frac{\Gamma(n \alpha+1)}{n !} \sin (n \pi \alpha), \quad \theta \in(0, \infty),
\end{aligned}
$$

$\xi_{\alpha}$ is a probability density function defined on $(0, \infty)$, that is,

$$
\xi_{\alpha}(\theta) \geq 0, \quad \theta \in(0, \infty) \quad \text { and } \quad \int_{0}^{\infty} \xi_{\alpha}(\theta) d \theta=1 .
$$

Lemma 2.2 [18] The operators $\mathcal{T}$ and \& have the following properties:

(i) For any fixed $t \geq 0, \mathcal{T}(t)$ and $s(t)$ are linear and bounded operators, i.e., for any $x \in X$,

$$
\|\mathcal{T}(t) x\| \leq M\|x\| \quad \text { and } \quad\|f(t) x\| \leq \frac{\alpha M}{\Gamma(1+\alpha)}\|x\| .
$$

(ii) $\{\mathcal{T}(t), t \geq 0\}$ and $\{f(t), t \geq 0\}$ are strongly continuous, which means that for every $x \in H$ and $0 \leq t^{\prime}<t^{\prime \prime} \leq T$, we have

$$
\left\|\mathcal{T}\left(t^{\prime \prime}\right) x-\mathcal{T}\left(t^{\prime}\right) x\right\| \rightarrow 0 \quad \text { and } \quad\left\|\S\left(t^{\prime \prime}\right) x-\S\left(t^{\prime}\right) x\right\| \rightarrow 0 \quad \text { as } t^{\prime} \rightarrow t^{\prime \prime} .
$$

(iii) For every $t>0, \mathcal{T}(t)$ and $\delta(t)$ are also compact operators if $T(t)$ is compact for every $t>0$.

In order to study the approximate controllability for the fractional control system (1), we introduce the following linear fractional differential system

$$
\left\{\begin{array}{l}
{ }^{c} D_{t}^{\alpha} x(t)=A x(t)+B u(t), \quad t \in J, \\
x(0)=x_{0} .
\end{array}\right.
$$


The controllability operator associated with (2) is defined by

$$
\Gamma_{0}^{T}=\int_{0}^{T}(T-s)^{\alpha-1} s(T-s) B B^{*} \varsigma^{*}(T-s) d s,
$$

where $B^{*}$ and $\delta^{*}$ denote the adjoint of $B$ and $\delta$, respectively.

Let $x(T ; \varphi, u)$ be the state value of $(1)$ at terminal time $T$, corresponding to the control $u$ and the initial value $\varphi$. Denote by $R(T, \varphi)=\left\{x(T ; \varphi, u): u \in L^{2}(J, U)\right\}$ the reachable set of system (1) at terminal time $T$, its closure in $H$ is denoted by $\overline{R(T, \varphi)}$.

Definition 2.4 The system (1) is said to be approximately controllable on $J$ if $\overline{R(T, \varphi)}=$ $L^{2}(\Omega, H)$.

Lemma 2.3 [19] The linear fractional control system (2) is approximately controllable on $J$ if and only if $\lambda\left(\lambda I+\Gamma_{0}^{T}\right) \rightarrow 0$ as $\lambda \rightarrow 0^{+}$in the strong operator topology.

Lemma 2.4 ([18] Krasnoselskii's fixed point theorem) Let $N$ be a Banach space, let $\hat{N}$ be a bounded closed and convex subset of $N$, and let $F_{1}, F_{2}$ be maps of $\hat{N}$ into $N$ such that $F_{1} x+F_{2} y \in \hat{N}$ for every pair $x, y \in \hat{N}$. If $F_{1}$ is a contraction and $F_{2}$ is completely continuous, then the equation $F_{1} x+F_{2} x=x$ has a solution on $\hat{N}$.

\section{Main results}

In this section, we formulate sufficient conditions for the approximate controllability of system (1). For this purpose, we first prove the existence of solutions for system (1). Second, in Theorem 3.2, we shall prove that system (1) is approximately controllable under certain assumptions. In order to prove our main results, we need the following assumptions.

(H1) The functions $f, h: J \times \mathcal{C}_{v} \rightarrow H$ are continuous, and there exist two positive constants $M_{f}$ and $M_{h}$ such that the function satisfies that

$$
E\|f(t, x)-f(t, y)\|^{2} \leq M_{f}\|x-y\|_{\mathcal{C}_{v}}^{2}, \quad E\|f(t, x)\|^{2} \leq M_{f}\left(1+\|x\|_{\mathcal{C}_{v}}^{2}\right)
$$

and

$$
E\|h(t, x)-h(t, y)\|^{2} \leq M_{h}\|x-y\|_{\mathcal{C}_{v}}^{2}, \quad E\|h(t, x)\|^{2} \leq M_{h}\left(1+\|x\|_{\mathcal{C}_{v}}^{2}\right)
$$

for every $x, y \in \mathcal{C}_{v}, t \in J$.

(H2) There exists a positive $M_{g}$ such that

$$
E\left\|g\left(t, x_{t}\right)-g\left(t, y_{t}\right)\right\|_{\mathcal{L}_{2}^{0}}^{2} \leq M_{g}\|x-y\|_{\mathcal{C}_{v}}^{2}, \quad E\left\|g\left(t, x_{t}\right)\right\|_{\mathcal{L}_{2}^{0}}^{2} \leq M_{g}\left(1+\|x\|_{\mathcal{C}_{v}}^{2}\right) .
$$

(H3) The function $I_{k}: H \rightarrow H$ is continuous, and there exists continuous nondecreasing function $L_{k}: R^{+} \rightarrow R^{+}$such that, for each $x \in H$,

$$
E\left\|I_{k}(x)\right\|^{2} \leq L_{k}\left(E\|x\|^{2}\right) \quad \text { and } \quad \liminf _{r \rightarrow \infty} \frac{L_{k}(r)}{r}=\beta_{k}<\infty, \quad k=1, \ldots, n .
$$

(H4) $\mu$ is continuous, and there exists some constant $M_{\mu}$ such that

$$
E\|\mu(x)\|^{2} \leq M_{\mu}\|x\|_{\mathcal{C}_{v}}^{2}
$$


(H5) The linear stochastic system (2) is approximately controllable on $[0, T]$.

The following lemma is required to define the control function.

Lemma 3.1 [6] For any $\bar{x}_{T} \in L^{2}\left(\mathcal{F}_{T}, H\right)$, there exists $\sigma(\cdot) \in L_{\mathscr{F}}^{2}\left(\Omega ; L^{2}\left(J ; L_{2}^{0}\right)\right)$ such that $\bar{x}_{T}=$ $E \bar{x}_{T}+\int_{0}^{T} \sigma(s) d W(s)$.

Now, for any $\lambda>0$ and $\bar{x}_{T} \in L^{2}\left(\mathcal{F}_{T}, H\right)$, we define the control function

$$
\begin{aligned}
u^{\lambda}(t)= & B^{*} \delta^{*}(T-t)\left(\lambda I+\Gamma_{0}^{T}\right)^{-1} \\
& \times\left[E \bar{x}_{T}+\int_{0}^{t} \sigma(s) d W(s)-\mathcal{T}(T)(\varphi(0)-\mu(x)-h(0, \varphi))-h\left(T, x_{T}\right)\right] \\
& -B^{*} \varsigma^{*}(T-t) \int_{0}^{t}\left(\lambda I+\Gamma_{s}^{T}\right)^{-1}(T-s)^{\alpha-1} \delta(T-s) f\left(s, x_{s}\right) d s \\
& -B^{*} \wp^{*}(T-t) \int_{0}^{t}\left(\lambda I+\Gamma_{s}^{T}\right)^{-1}(T-s)^{\alpha-1} \delta(T-s) g\left(s, x_{s}\right) d W(s) \\
& -B^{*} \varsigma^{*}(T-t)\left(\lambda I+\Gamma_{0}^{T}\right)^{-1} \sum_{0<\tau_{k}<T} \mathcal{T}\left(T-\tau_{k}\right) I_{k}\left(x\left(\tau_{k}^{-}\right)\right) .
\end{aligned}
$$

Theorem 3.1 Assume that the assumptions (H1)-(H4) hold. Then for each $\lambda>0$, the system (1) has a mild solution on $[0, T]$, provided that

$$
\begin{aligned}
& {\left[8 l^{2} M^{2} M_{\mu}+4 l^{2} M_{h}+4 l^{2} M_{f}\left(\frac{M T^{\alpha}}{\Gamma(1+\alpha)}\right)^{2}\right.} \\
& \left.\quad+4 l^{2} M_{g} \frac{T^{2 \alpha-1}}{2 \alpha-1}\left(\frac{\alpha M}{\Gamma(1+\alpha)}\right)^{2}+4 l^{2} n M^{2} \sum_{k=1}^{n} \beta_{k}\right] \times\left[6+\frac{48 T^{2 \alpha}}{\lambda^{2} \alpha^{2}}\left(\frac{\alpha M M_{B}}{\Gamma(1+\alpha)}\right)^{4}\right] \leq 1
\end{aligned}
$$

and

$$
L=3 l^{2}\left[M_{h}+M_{f} \frac{T^{2 \alpha}}{\alpha^{2}}\left(\frac{\alpha M}{\Gamma(1+\alpha)}\right)^{2}+M_{g} \frac{T^{2 \alpha-1}}{2 \alpha-1}\left(\frac{\alpha M}{\Gamma(1+\alpha)}\right)^{2}\right]<1 .
$$

Proof For any $\lambda>0$, define the operator $\Phi: \mathcal{B}_{T} \rightarrow \mathcal{B}_{T}$ by

$$
(\Phi x)(t)=\left\{\begin{array}{l}
\varphi(t), \quad t \in(-\infty, 0] \\
\mathcal{T}(t)[\varphi(0)-\mu(x)-h(0, \varphi)]+h\left(t, x_{t}\right) \\
\quad+\int_{0}^{t}(t-s)^{\alpha-1} s(t-s) B u^{\lambda}(s) d s \\
\quad+\int_{0}^{t}(t-s)^{\alpha-1} s(t-s) f\left(s, x_{s}\right) d s \\
\quad+\int_{0}^{t}(t-s)^{\alpha-1} s(t-s) g\left(s, x_{s}\right) d W(s) \\
\quad+\sum_{0<\tau_{k}<t} \mathcal{T}\left(t-\tau_{k}\right) I_{k}\left(x\left(\tau_{k}^{-}\right)\right), \quad t \in J .
\end{array}\right.
$$

We shall show that the operator $\Phi$ has a fixed point in the space $\mathcal{B}_{T}$, which is the mild solution of (1). Let $x(t)=z(t)+\hat{\varphi}(t),-\infty<t \leq T$, where $\hat{\varphi}(t)$ is defined by

$$
\hat{\varphi}(t)= \begin{cases}\varphi(t), & t \in(-\infty, 0] \\ \mathcal{T}(t) \varphi(0), & t \in J\end{cases}
$$


Then $\hat{\varphi}(t) \in \mathcal{B}_{T}$, and it is clear that $x$ satisfies (1) if and only if $z$ satisfies $z_{0}=0$ and

$$
\begin{aligned}
z(t)= & \mathcal{T}(t)[-\mu(z+\hat{\varphi})-h(0, \varphi)]+h\left(t, z_{t}+\hat{\varphi}_{t}\right) \\
& +\int_{0}^{t}(t-s)^{\alpha-1} s(t-s) B u^{\lambda}(s) d s \\
& +\int_{0}^{t}(t-s)^{\alpha-1} s(t-s) f\left(s, z_{s}+\hat{\varphi}_{s}\right) d s \\
& +\int_{0}^{t}(t-s)^{\alpha-1} s(t-s) g\left(s, z_{s}+\hat{\varphi}_{s}\right) d W(s) \\
& +\sum_{0<\tau_{k}<t} \mathcal{T}\left(t-\tau_{k}\right) I_{k}\left(z\left(\tau_{k}^{-}\right)+\hat{\varphi}\left(\tau_{k}^{-}\right)\right), \quad t \in J .
\end{aligned}
$$

Set $\mathcal{B}_{T}^{0}=\left\{z \in \mathcal{B}_{T}, z_{0}=0 \in \mathcal{C}_{v}\right\}$, and for any $z \in \mathcal{B}_{T}^{0}$, we define

$$
\begin{aligned}
\|z\|_{\mathcal{B}_{T}^{0}} & =\left\|z_{0}\right\|_{\mathcal{C}_{h}}+\sup _{s \in[0, T]}\left(E\|z(s)\|^{2}\right)^{\frac{1}{2}} \\
& =\sup _{s \in[0, T]}\left(E\|z(s)\|^{2}\right)^{\frac{1}{2}}, \quad x \in \mathcal{B}_{T} .
\end{aligned}
$$

Thus, $\left(\mathcal{B}_{T}^{0},\|\cdot\|_{\mathcal{B}_{T}^{0}}\right)$ is a Banach space. Let $B_{r}=\left\{z \in \mathcal{B}_{T}^{0}:\|z\|_{\mathcal{B}_{T}^{0}}^{2} \leq r\right\}$ for some $r>0$, then $B_{r}$, for each $r$, is a bounded, closed subset of $H$. Moreover, for $z \in B_{r}$, by lemma 2.1, we have

$$
\begin{aligned}
\left\|z_{t}+\hat{\varphi}_{t}\right\|_{\mathcal{C}_{v}}^{2} & \leq 2\left(\left\|z_{t}\right\|_{\mathcal{C}_{v}}^{2}+\left\|\hat{\varphi}_{t}\right\|_{\mathcal{C}_{v}}^{2}\right) \\
& \leq 4\left(l^{2} \sup _{0 \leq s \leq t} E\|z(s)\|^{2}+\left\|z_{0}\right\|_{\mathcal{C}_{v}}^{2}+l^{2} \sup _{0 \leq s \leq t} E\|\hat{\varphi}(s)\|^{2}+\left\|\hat{\varphi}_{0}\right\|_{\mathcal{C}_{v}}^{2}\right) \\
& \leq 4 l^{2}\left(r+M^{2} E\|\varphi(0)\|_{H}^{2}\right)+4\|\varphi\|_{\mathcal{C}_{v}}^{2} .
\end{aligned}
$$

For the sake of convenience, we divide the proof into several steps.

Step 1. We claim that there exists a positive number $r$ such that $\Phi\left(B_{r}\right) \subset B_{r}$. If this is not true, then, for each positive integer $r$, there exists $z^{r} \in B_{r}$ such that $E\left\|\Phi\left(z^{r}\right)(t)\right\|^{2}>r$ for $t \in(-\infty, T], t$ may depending upon $r$. However, on the other hand, we have

$$
\begin{aligned}
r \leq & E\left\|\Phi\left(z^{r}\right)(t)\right\|^{2} \\
\leq & 6 E\left\|\mathcal{T}(t)\left[-\mu\left(z^{r}+\hat{\varphi}\right)-h(0, \varphi)\right]\right\|^{2}+6 E\left\|h\left(t, z_{t}^{r}+\hat{\varphi}_{t}\right)\right\|^{2} \\
& +6 E\left\|\int_{0}^{t}(t-s)^{\alpha-1} s(t-s) B u^{\lambda}(s) d s\right\|^{2} \\
& +6 E\left\|\int_{0}^{t}(t-s)^{\alpha-1} s(t-s) f\left(s, z_{s}^{r}+\hat{\varphi}_{s}\right) d s\right\|^{2} \\
& +6 E\left\|\int_{0}^{t}(t-s)^{\alpha-1} s(t-s) g\left(s, z_{s}^{r}+\hat{\varphi}_{s}\right) d W(s)\right\|^{2} \\
& +6 E\left\|\sum_{0<\tau_{k}<t} \mathcal{T}\left(t-\tau_{k}\right) I_{k}\left(z\left(\tau_{k}^{-}\right)+\hat{\varphi}\left(\tau_{k}^{-}\right)\right)\right\|^{2}, \quad t \in J .
\end{aligned}
$$


By using (H1)-(H4), Lemma 2.1 and Hölder's inequality, we obtain

$$
\begin{aligned}
r \leq & E\left\|\Phi\left(z^{r}\right)(t)\right\|^{2} \\
\leq & 12 M^{2} M_{\mu}\left\|z^{r}+\hat{\varphi}\right\|_{\mathcal{C}_{v}}^{2}+12 M^{2} M_{h}\left(1+\|\varphi\|_{\mathcal{C}_{v}}^{2}\right)+6 M_{h}\left(1+\left\|\left(z_{t}^{r}+\hat{\varphi}_{t}\right)\right\|_{\mathcal{C}_{v}}^{2}\right) \\
& +6 \frac{T^{\alpha}}{\alpha}\left(\frac{\alpha M M_{B}}{\Gamma(1+\alpha)}\right)^{2} \int_{0}^{t}(t-s)^{\alpha-1} E\left\|u^{\lambda}(s)\right\|^{2} d s \\
& +6 \frac{T^{\alpha}}{\alpha}\left(\frac{\alpha M}{\Gamma(1+\alpha)}\right)^{2} \int_{0}^{t}(t-s)^{\alpha-1} E\left\|f\left(s, z_{s}^{r}+\hat{\varphi}_{s}\right)\right\|^{2} d s \\
& +6\left(\frac{\alpha M}{\Gamma(1+\alpha)}\right)^{2} \int_{0}^{t}(t-s)^{2(\alpha-1)} E\left\|g\left(s, z_{s}^{r}+\hat{\varphi}_{s}\right)\right\|_{\mathcal{L}_{2}^{0}}^{2} d s \\
& +6 n M^{2} \sum_{k=1}^{n} E\left\|I_{k}\left(z\left(\tau_{k}^{-}\right)+\hat{\varphi}\left(\tau_{k}^{-}\right)\right)\right\|^{2} \\
\leq & 12 M^{2} M_{\mu} r^{\prime}+12 M^{2} M_{h}\left(1+\|\varphi\|_{\mathcal{C}_{v}}^{2}\right)+6 M_{h}\left(1+r^{\prime}\right)+\frac{48 T^{2 \alpha}}{\lambda^{2} \alpha^{2}}\left(\frac{\alpha M M_{B}}{\Gamma(1+\alpha)}\right)^{4} M_{C} \\
& +6\left(\frac{M T^{\alpha}}{\Gamma(1+\alpha)}\right)^{2} M_{f}\left(1+r^{\prime}\right)+6 \frac{T^{2 \alpha-1}}{2 \alpha-1}\left(\frac{\alpha M}{\Gamma(1+\alpha)}\right)^{2} M_{g}\left(1+r^{\prime}\right) \\
& +6 n M^{2} \sum_{k=1}^{n} L_{k}\left(r^{\prime}\right),
\end{aligned}
$$

where $r^{\prime}=4 l^{2}\left(r+M^{2} E\|\varphi(0)\|_{H}^{2}\right)+4\|\varphi\|_{\mathcal{C}_{v}}^{2},\|B\| \leq M_{B}$, and

$$
\begin{aligned}
M_{C}= & 2 E\left\|\bar{x}_{T}\right\|^{2}+2 \int_{0}^{T} E\|\sigma(s)\|_{\mathcal{L}_{2}^{0}}^{2} d s+M^{2}\|\varphi\|_{\mathcal{C}_{v}}^{2}+M^{2} M_{\mu}\left(1+r^{\prime}\right) \\
& +M^{2} M_{h}\left(1+\|\varphi\|_{\mathcal{C}_{v}}^{2}\right)+M_{h}\left(1+r^{\prime}\right)+\left(\frac{M T^{\alpha}}{\Gamma(1+\alpha)}\right)^{2} M_{f}\left(1+r^{\prime}\right) \\
& +\frac{T^{2 \alpha-1}}{2 \alpha-1}\left(\frac{\alpha M}{\Gamma(1+\alpha)}\right)^{2} M_{g}\left(1+r^{\prime}\right)+n M^{2} \sum_{k=1}^{n} L_{k}\left(r^{\prime}\right) .
\end{aligned}
$$

Dividing both sides by $r$ and taking the limit as $r \rightarrow \infty$, we obtain

$$
\begin{aligned}
1 \leq & {\left[8 l^{2} M^{2} M_{\mu}+4 l^{2} M_{h}+4 l^{2} M_{f}\left(\frac{M T^{\alpha}}{\Gamma(1+\alpha)}\right)^{2}\right.} \\
& \left.+4 l^{2} M_{g} \frac{T^{2 \alpha-1}}{2 \alpha-1}\left(\frac{\alpha M}{\Gamma(1+\alpha)}\right)^{2}+4 l^{2} n M^{2} \sum_{k=1}^{n} \beta_{k}\right] \cdot\left[6+\frac{48 T^{2 \alpha}}{\lambda^{2} \alpha^{2}}\left(\frac{\alpha M M_{B}}{\Gamma(1+\alpha)}\right)^{4}\right],
\end{aligned}
$$

which is a contradiction to our assumption. Thus, for each $\lambda>0$, there exists some positive number $r$ such that $\Phi\left(B_{r}\right) \subset B_{r}$.

Next, we show that the operator $\Phi$ is condensing, for convenience, we decompose $\Phi$ as $\Phi=\Phi_{1}+\Phi_{2}$, where

$$
\begin{aligned}
\left(\Phi_{1} z\right)(t)= & h\left(t, z_{t}+\hat{\varphi}_{t}\right)+\int_{0}^{t}(t-s)^{\alpha-1} \delta(t-s) f\left(s, z_{s}+\hat{\varphi}_{s}\right) d s \\
& +\int_{0}^{t}(t-s)^{\alpha-1} \delta(t-s) g\left(s, z_{s}+\hat{\varphi}_{s}\right) d W(s)
\end{aligned}
$$




$$
\begin{aligned}
\left(\Phi_{2} z\right)(t)= & \mathcal{T}(t)[-\mu(z+\hat{\varphi})-h(0, \varphi)]+\int_{0}^{t}(t-s)^{\alpha-1} s(t-s) B u^{\lambda}(s) d s . \\
& +\sum_{0<\tau_{k}<t} \mathcal{T}\left(t-\tau_{k}\right) I_{k}\left(z\left(\tau_{k}^{-}\right)+\hat{\varphi}\left(\tau_{k}^{-}\right)\right), \quad t \in J .
\end{aligned}
$$

Step 2. We prove that $\Phi_{1}$ is a contraction on $B_{r}$. Let $t \in J$ and $z_{1}, z_{2} \in B_{r}$, we have

$$
\begin{aligned}
E \| & \Phi_{1} z_{1}(t)-\Phi_{1} z_{2}(t) \|^{2} \\
\leq & 3 E\left\|h\left(t, z_{1, t}+\hat{\varphi}_{t}\right)-h\left(t, z_{2, t}+\hat{\varphi}_{t}\right)\right\|^{2} \\
& +3 E\left\|\int_{0}^{t}(T-s)^{\alpha-1} s(T-s)\left[f\left(s, z_{1, s}+\hat{\varphi}_{s}\right)-f\left(s, z_{2, s}+\hat{\varphi}_{s}\right)\right] d s\right\|^{2} \\
& +3 E\left\|\int_{0}^{t}(T-s)^{\alpha-1} s(T-s)\left[g\left(s, z_{1, s}+\hat{\varphi}_{s}\right)-g\left(s, z_{2, s}+\hat{\varphi}_{s}\right)\right] d W(s)\right\|^{2} \\
\leq & 3 M_{h}\left\|z_{1, t}-z_{2, t}\right\|_{\mathcal{C}_{v}}^{2}+3 M_{f} \frac{T^{\alpha}}{\alpha}\left(\frac{\alpha M}{\Gamma(1+\alpha)}\right)^{2} \int_{0}^{t}(T-s)^{\alpha-1}\left\|z_{1, s}-z_{2, s}\right\|_{\mathcal{C}_{v}}^{2} d s \\
& +3 M_{g}\left(\frac{\alpha M}{\Gamma(1+\alpha)}\right)^{2} \int_{0}^{t}(T-s)^{2(\alpha-1)}\left\|z_{1, s}-z_{2, s}\right\|_{\mathcal{C}_{v}}^{2} d s \\
\leq & L \sup _{s \in J} E\left\|z_{1}(s)-z_{2}(s)\right\|^{2},
\end{aligned}
$$

where $L=3 l^{2}\left[M_{h}+M_{f} \frac{T^{2 \alpha}}{\alpha^{2}}\left(\frac{\alpha M}{\Gamma(1+\alpha)}\right)^{2}+M_{g} \frac{T^{2 \alpha-1}}{2 \alpha-1}\left(\frac{\alpha M}{\Gamma(1+\alpha)}\right)^{2}\right]<1$, hence $\Phi_{1}$ is a contraction.

Step 3. $\Phi_{2}$ maps bounded sets into bounded sets in $B_{r}$,

$$
\begin{aligned}
E\left\|\Phi_{2} z(t)\right\|_{H}^{2} \leq & 3 E\|\mathcal{T}(t)[-\mu(z+\hat{\varphi})-h(0, \varphi)]\|^{2} \\
& +3 E\left\|\int_{0}^{t}(t-s)^{\alpha-1} \delta(t-s) B u^{\lambda}(s) d s\right\|^{2} \\
& +3 E\left\|\sum_{0<\tau_{k}<t} \mathcal{T}\left(t-\tau_{k}\right) I_{k}\left(z\left(\tau_{k}^{-}\right)+\hat{\varphi}\left(\tau_{k}^{-}\right)\right)\right\|^{2} \\
\leq & 6 M^{2} M_{\mu} r^{\prime}+6 M^{2} M_{h}\left(1+\|\varphi\|^{2}\right) \\
& +\frac{24}{\lambda^{2}} \frac{T^{2 \alpha}}{\alpha^{2}}\left(\frac{\alpha M M_{B}}{\Gamma(1+\alpha)}\right)^{4} M_{C}+3 M^{2} n^{2} \sum_{k=1}^{n} L_{k}\left(r^{\prime}\right)
\end{aligned}
$$

$:=\Lambda$.

Therefore, for each $z \in B_{r}$, we get $E\left\|\Phi_{2} z(t)\right\|^{2} \leq \Lambda$.

Step 4. The map $\Phi_{2}$ is equicontinuous. Let $0<t_{1}<t_{2} \leq T$ and $t_{1}, t_{2} \in J \backslash\left\{\tau_{1}, \tau_{2}, \ldots, \tau_{n}\right\}$.

Then, we have

$$
\begin{aligned}
E\left\|\Phi_{2} z\left(t_{2}\right)-\Phi_{2} z\left(t_{1}\right)\right\|^{2} \leq & 5 E\left\|\left[\mathcal{T}\left(t_{2}\right)-\mathcal{T}\left(t_{1}\right)\right][-\mu(z+\hat{\varphi})-h(0, \varphi)]\right\|^{2} \\
& +5 E\left\|\int_{0}^{t_{1}}\left(t_{1}-s\right)^{\alpha-1}\left[s\left(t_{2}-s\right)-\varsigma\left(t_{1}-s\right)\right] B u^{\lambda}(s) d s\right\|^{2} \\
& +5 E\left\|\int_{0}^{t_{1}}\left[\left(t_{2}-s\right)^{\alpha-1}-\left(t_{1}-s\right)^{\alpha-1}\right] s\left(t_{2}-s\right) B u^{\lambda}(s) d s\right\|^{2}
\end{aligned}
$$




$$
\begin{aligned}
& +5 E\left\|\int_{t_{1}}^{t_{2}}\left(t_{2}-s\right)^{\alpha-1} \delta\left(t_{2}-s\right) B u^{\lambda}(s) d s\right\|^{2} \\
& +5 E\left\|\sum_{0<\tau_{k}<T}\left[\mathcal{T}\left(t_{2}-\tau_{k}\right)-\mathcal{T}\left(t_{1}-\tau_{k}\right)\right] I_{k}\left(z\left(\tau_{k}^{-}\right)+\hat{\varphi}\left(\tau_{k}^{-}\right)\right)\right\|^{2} .
\end{aligned}
$$

Noting the fact that for every $\epsilon>0$, there exists a $\delta>0$ such that, whenever $\left|s_{1}-s_{2}\right|<\delta$ for every $s_{1}, s_{2} \in J,\left\|\mathcal{T}\left(s_{1}\right)-\mathcal{T}\left(s_{2}\right)\right\|<\epsilon$ and $\left\|\delta\left(s_{1}\right)-\delta\left(s_{2}\right)\right\|<\epsilon$. Therefore, when $\left|t_{2}-t_{1}\right|<\delta$, we have

$$
\begin{aligned}
E\left\|\Phi_{2} z\left(t_{2}\right)-\Phi_{2} z\left(t_{1}\right)\right\|^{2} \leq & 10 \epsilon^{2}\left[M_{\mu} r^{\prime}+M_{h}\left(1+\|\varphi\|^{2}\right)\right]+\frac{40 \epsilon^{2} M_{B}^{2}}{\lambda^{2}} \frac{T^{2 \alpha}}{\alpha^{2}} M_{C} \\
& +\frac{40 M_{C}}{\alpha^{2} \lambda^{2}}\left(\frac{\alpha M M_{B}}{\Gamma(\alpha+1)}\right)^{4}\left[t_{2}^{\alpha}-t_{1}^{\alpha}-\left(t_{2}-t_{1}\right)^{\alpha}\right]^{2} \\
& +\frac{40 M_{C}}{\alpha^{2} \lambda^{2}}\left(\frac{\alpha M M_{B}}{\Gamma(\alpha+1)}\right)^{4}\left(t_{2}-t_{1}\right)^{2 \alpha}+5 n \epsilon^{2} \sum_{k=1}^{n} L_{k}\left(r^{\prime}\right) .
\end{aligned}
$$

The right hand of the inequality above tends to 0 as $t_{2} \rightarrow t_{1}$ and $\epsilon \rightarrow 0$, hence the set $\left\{\Phi_{2} z, z \in B_{r}\right\}$ is equicontinuous.

Step 5. The set $V(t)=\left\{\Phi_{2} z(t), z \in B_{r}\right\}$ is relatively compact in $B_{r}$. Let $0<t \leq T$ be fixed and $0<\epsilon<t$. For $\delta>0, z \in B_{r}$, we define

$$
\begin{aligned}
\Phi_{2}^{\epsilon, \delta} z(t)= & \int_{\delta}^{\infty} \xi_{\alpha}(\theta) T\left(t^{\alpha} \theta\right)[-\mu(z+\hat{\varphi})-h(0, \varphi)] d \theta \\
& +\alpha \int_{0}^{t-\epsilon} \int_{\delta}^{\infty} \theta(t-s)^{\alpha-1} \xi_{\alpha}(\theta) T\left((t-s)^{\alpha} \theta\right) B u^{\lambda}(s) d \theta d s . \\
& +\sum_{0<\tau_{k}<t} \int_{\delta}^{\infty} \xi_{\alpha}(\theta) T\left(\left(t-\tau_{k}\right)^{\alpha} \theta\right) I_{k}\left(z\left(\tau_{k}^{-}\right)+\hat{\varphi}\left(\tau_{k}^{-}\right)\right) d \theta \\
= & T\left(\epsilon^{\alpha} \delta\right) \int_{\delta}^{\infty} \xi_{\alpha}(\theta) T\left(t^{\alpha} \theta-\epsilon^{\alpha} \delta\right)[-\mu(z+\hat{\varphi})-h(0, \varphi)] d \theta \\
& +\alpha T\left(\epsilon^{\alpha} \delta\right) \int_{0}^{t-\epsilon} \int_{\delta}^{\infty} \theta(t-s)^{\alpha-1} \xi_{\alpha}(\theta) T\left((t-s)^{\alpha} \theta-\epsilon^{\alpha} \delta\right) B u(s) d \theta d s . \\
& +\sum_{0<\tau_{k}<t} T\left(\epsilon^{\alpha} \delta\right) \int_{\delta}^{\infty} \xi_{\alpha}(\theta) T\left(\left(t-\tau_{k}\right)^{\alpha} \theta-\epsilon^{\alpha} \delta\right) I_{k}\left(z\left(\tau_{k}^{-}\right)+\hat{\varphi}\left(\tau_{k}^{-}\right)\right) d \theta .
\end{aligned}
$$

Then from the compactness of $T\left(\epsilon^{\alpha} \delta\right)$, we obtain that $V_{\epsilon, \delta}(t)=\left\{\Phi_{2}^{\epsilon, \delta} z(t): z \in B_{r}\right\}$ is relatively compact in $H$ for every $\epsilon, 0<\epsilon<t$. Moreover, for $z \in B_{r}$, we can easily prove that $\Phi_{2}^{\epsilon, \delta} z(t)$ is convergent to $\Phi_{2} z(t)$ in $B_{r}$ as $\epsilon \rightarrow 0$ and $\delta \rightarrow 0$, hence the set $V(t)=\left\{\Phi_{2} z(t), z \in\right.$ $B_{r}$ \} is also relatively compact in $B_{r}$. Thus, by Arzela-Ascoli theorem $\Phi_{2}$ is completely continuous. Consequently, from Lemma 2.4, $\Phi$ has a fixed point, which is a mild solution of (1).

Theorem 3.2 Assume that (H1)-(H5) are satisfied, and the conditions of Theorem 3.1 hold. Further, if the functions $f$ and $g$ are uniformly bounded, and $T(t)$ is compact, then the system (1) is approximately controllable on $[0, T]$. 
Proof Let $x^{\lambda}$ be a solution of (1), then we can easily get that

$$
\begin{aligned}
x^{\lambda}(T)= & \bar{x}_{T}-\lambda\left(\lambda I+\Gamma_{0}^{T}\right)^{-1}\left[E \bar{x}_{T}+\int_{0}^{T} \sigma(s) d W(s)\right. \\
& \left.-\mathcal{T}(T)(\varphi(0)-\mu(x)-h(0, \varphi))-h\left(T, x_{T}^{\lambda}\right)\right] \\
& +\lambda \int_{0}^{T}\left(\lambda I+\Gamma_{s}^{T}\right)^{-1}(T-s)^{\alpha-1} \delta(T-s) f\left(s, x_{s}^{\lambda}\right) d s \\
& +\lambda \int_{0}^{T}\left(\lambda I+\Gamma_{s}^{T}\right)^{-1}(T-s)^{\alpha-1} \delta(T-s) g\left(s, x_{s}^{\lambda}\right) d W(s) \\
& +\lambda\left(\lambda I+\Gamma_{0}^{T}\right)^{-1} \sum_{0<\tau_{k}<T} \mathcal{T}\left(T-\tau_{k}\right) I_{k}\left(x_{s}^{\lambda}\right) .
\end{aligned}
$$

In view of the assumptions that $f$ and $g$ are uniformly bounded on $J$, hence, there is a subsequence still denoted by $f\left(s, x_{s}^{\lambda}\right)$ and $g\left(s, x_{s}^{\lambda}\right)$, which converges weakly to say $f(s)$ in $H$, and $g(s)$ in $\mathcal{L}(U, H)$. On the other hand, by assumption (H5), the operator $\lambda\left(\lambda I+\Gamma_{s}^{T}\right)^{-1} \rightarrow 0$ strongly as $\lambda \rightarrow 0^{+}$for all $0 \leq s \leq T$, and, moreover, $\left\|\lambda\left(\lambda I+\Gamma_{s}^{T}\right)^{-1}\right\| \leq 1$. Thus, the Lebesgue dominated convergence theorem and the compactness of $\delta$ yield

$$
\begin{aligned}
E\left\|x^{\lambda}(T)-\bar{x}_{T}\right\|^{2} \leq & 4\left\|\lambda\left(\lambda I+\Gamma_{0}^{T}\right)^{-1}\right\|^{2} E \| \bar{x}_{T}+\int_{0}^{T} \sigma(s) d W(s) \\
& -\mathcal{T}(T)(\varphi(0)-\mu(x)-h(0, \varphi))-h\left(T, x_{T}^{\lambda}\right) \|^{2} \\
& +4 E\left(\int_{0}^{T}\left\|\lambda\left(\lambda I+\Gamma_{s}^{T}\right)^{-1}(T-s)^{\alpha-1} s(T-s) f\left(s, x_{s}^{\lambda}\right)\right\| d s\right)^{2} \\
& +4 E\left\|\int_{0}^{T} \lambda\left(\lambda I+\Gamma_{s}^{T}\right)^{-1}(T-s)^{\alpha-1} s(T-s) g\left(s, x_{s}^{\lambda}\right) d W(s)\right\|^{2} \\
& +4\left\|\lambda\left(\lambda I+\Gamma_{0}^{T}\right)^{-1}\right\|^{2} E\left\|\sum_{0<\tau_{k}<T} \mathcal{T}\left(T-\tau_{k}\right) I_{k}\left(x_{s}^{\lambda}\right)\right\|^{2} \rightarrow 0, \quad \text { as } \lambda \rightarrow 0^{+} .
\end{aligned}
$$

This gives the approximate controllability of (1), the proof is complete.

\section{An example}

As an application, we consider an impulsive neutral stochastic partial differential equation with the following form

$$
\left\{\begin{array}{l}
{ }^{c} D_{t}^{\alpha}\left[x(t, y)-\int_{-\infty}^{t} e^{4(s-t)} x(s, y) d s\right] \\
\quad=\frac{\partial^{2}}{\partial y^{2}}\left[x(t, y)-\int_{-\infty}^{t} e^{4(s-t)} x(s, y) d s\right] \\
\quad+b(y) u(t)+\int_{0}^{T} k(t, s) \int_{-\infty}^{s} \alpha(s-\theta) x(\theta, y) d \theta d s \\
\quad+\int_{-\infty}^{t} \alpha(t-s) x(s, y) d W(s), \quad x \in[0, \pi], t \in J=[0, T], \\
x\left(\tau_{k}^{+}, y\right)-x\left(\tau_{k}^{-}, y\right)=I_{k}\left(x\left(\tau_{k}^{-}, y\right)\right), \quad k=1,2, \ldots, n, \\
x(t, 0)=x(t, \pi)=0, \quad t \in J=[0, T], \\
x(0, y)+\int_{0}^{\pi} k_{1}(y, z) x(t, z) d z=\varphi(t, y), \quad t \in(-\infty, 0] .
\end{array}\right.
$$


Let $U=H=L^{2}([0, \pi])$ and $v(t)=e^{2 t}, t<0$ with $l=\frac{1}{2}$. To study the approximate controllability of (3), assume that $k(t, s)$ is measurable and continuous on $[0, T] \times[0, T]$ and thus bounded by $L_{k} \cdot \alpha(t)$ is measurable and continuous with finite $L_{\alpha}^{2}=\int_{-\infty}^{0} \frac{\alpha^{2}(-s)}{v(s)} d s$.

We define the operator $A$ by $A x=\frac{\partial^{2} x}{\partial y^{2}}$ with domain $D(A)=\left\{x \in H, \frac{\partial x}{\partial y}, \frac{\partial^{2} x}{\partial y^{2}} \in H\right.$ and $x(0)=$ $x(\pi)=0\}$. It is well known that $A$ generates an analytic semigroup $T(t), t \geq 0$ given by $T(t) x=\sum_{n=1}^{\infty} e^{-n^{2} t}\left\langle x, e_{n}\right\rangle e_{n}, x \in H$, and $e_{n}(y)=(2 / \pi)^{1 / 2} \sin (n y), n=1,2, \ldots$, is the orthogonal set of eigenvectors of $A$.

Define the operators $h, f: J \times \mathcal{C}_{v} \rightarrow H, g: J \times \mathcal{C}_{v} \rightarrow L_{2}^{0}(U, H)$ by

$$
\begin{aligned}
& h(t, \varphi)(y)=\int_{-\infty}^{0} e^{-4 s} \varphi(s)(y) d s, \\
& f(\varphi)(y)=\int_{0}^{T} k(t, s) \int_{-\infty}^{0} \alpha(-\theta) \varphi(\theta, y) d \theta d s, \\
& g(\varphi)(y)=\int_{-\infty}^{0} \alpha(-s) \varphi(s, y) d W(s) .
\end{aligned}
$$

With the choice of $A, h, f, g$, (3) can be rewritten as the abstract form of system (1). Thus, under the appropriate conditions on the functions $h, f, g$ and $I_{k}$ as those in (H1)-(H5), system (3) is approximately controllable.

\section{Competing interests}

The authors declare that they have no competing interests.

\section{Authors' contributions}

All authors contributed equally to the manuscript.

\section{Acknowledgements}

We are very grateful to the anonymous referee and the associate editor for their careful reading and helpful comments. This work was substantially supported by the National Natural Sciences Foundation of China (No. 11071259), Research Fund for the Doctoral Program of Higher Education of China (No. 20110162110060).

Received: 16 April 2013 Accepted: 12 August 2013 Published: 28 August 2013

\section{References}

1. Kilbas, AA, Srivastava, HM, Trujillo, JJ: Theory and Applications of Fractional Differential Equations. North Holland Mathematics Studies, vol. 204. Elsevier, Amsterdam (2006)

2. Miller, KS, Ross, B: An Introduction to the Fractional Calculus and Differential Equations. Wiley, New York (1993)

3. Podlubny, I: Fractional Differential Equations. Academic Press, San Diego (1999)

4. Benchohra, M, Henderson, J, Ntouyas, SK, Ouahab, A: Existence results for fractional order functional differential equations with infinite delay. J. Math. Anal. Appl. 338, 1340-1350 (2008)

5. Zhou, Y, Jiao, F, Li, J: Existence and uniqueness for fractional neutral differential equations with infinite delay. Nonlinear Anal. 71, 3249-3256 (2009)

6. Mahmudov, NI: Controllability of linear stochastic systems in Hilbert spaces. J. Math. Anal. Appl. 259, 64-82 (2001)

7. Dauer, JP, Mahmudov, NI: Controllability of stochastic semilinear functional differential equations in Hilbert spaces. J. Math. Anal. Appl. 290, 373-394 (2004)

8. Park, JY, Balasubramaniam, P, Kumaresan, N: Controllability for neutral stochastic functional integrodifferential infinite delay systems in abstract space. Numer. Funct. Anal. Optim. 28, 1369-1386 (2007)

9. Li, CX, Sun, JT, Sun, RY: Stability analysis of a class of stochastic differential delay equations with nonlinear impulsive effects. J. Franklin Inst. 347, 1186-1198 (2010)

10. Sakthivel, R, Mahmudov, NI, Lee, SG: Controllability of non-linear impulsive stochastic systems. Int. J. Control 82 , 801-807 (2009)

11. Shen, LJ, Shi, JP, Sun, JT: Complete controllability of impulsive stochastic integro-differential systems. Automatica 46 1068-1073 (2010)

12. Shen, LJ, Sun, JT: Approximate controllability of stochastic impulsive functional systems with infinite delay. Automatica 48, 2705-2709 (2012)

13. Subalakshmi, R, Balachandran, K: Approximate controllability of nonlinear stochastic impulsive intergrodifferential systems in Hilbert spaces. Chaos Solitons Fractals 42, 2035-2046 (2009)

14. Da Prato, G, Zabczyk, J: Stochastic Equations in Infinite Dimensions. Cambridge University Press, Cambridge (1992)

15. Ren, Y, Sun, DD: Second-order neutral stochastic evolution equations with infinite delay under Caratheodory conditions. J. Optim. Theory Appl. 147, 569-582 (2010) 
16. Ren, Y, Zhou, Q, Chen, L: Existence, uniqueness and stability of mild solutions for time-dependent stochastic evolution equations with Poisson jumps and infinite delay. J. Optim. Theory Appl. 149, 315-331 (2011)

17. Chang, YK: Controllability of impulsive functional differential systems with infinite delay in Banach space. Chaos Solitons Fractals 33, 1601-1609 (2007)

18. Zhou, Y, Jiao, F: Existence of mild solution for fractional neutral evolution equations. Comput. Math. Appl. 59, 1063-1077 (2010)

19. Mahmudov, NI, Denker, A: On controllability of linear stochastic systems. Int. J. Control 73, 144-151 (2000)

doi:10.1186/1687-2770-2013-193

Cite this article as: Zang and Li: Approximate controllability of fractional impulsive neutral stochastic differential equations with nonlocal conditions. Boundary Value Problems 2013 2013:193.

Submit your manuscript to a SpringerOpen ${ }^{\circ}$ journal and benefit from:

- Convenient online submission

- Rigorous peer review

- Immediate publication on acceptance

- Open access: articles freely available online

- High visibility within the field

- Retaining the copyright to your article 